\title{
Effect of endmember clustering on proportion estimation: Results on the Share 2012 dataset
}

\author{
Erdinc Gunes and Seniha Esen Yuksel \\ Hacettepe University, Department of Electrical and Electronics Engineering \\ Beytepe, Ankara, Turkey \\ Contact: egunes@aselsan.com.tr, eyuksel@ee.hacettepe.edu.tr
}

\begin{abstract}
Estimating the number of endmembers and their spectrum is a challenging task. For one, endmember detection algorithms may over or underestimate the number of endmembers in a given scene. Further, even if the number of endmembers are known beforehand, result of the endmember detection algorithms may not be accurate. They may find multiple endmembers representing the same class, while completely missing some of the endmembers representing the other classes. This hinders the performance of unmixing, resulting in incorrect endmember proportion estimates. In this study, SHARE-2012 AVON data pertaining to the unmixing experiment was considered. It was cropped to include only the eight pieces of cloth and a portion of the surrounding asphalt and grass. This data was used to evaluate the performance of five endmember detection algorithms, namely the PPI, VCA, N-FINDR, ICE and SPICE; none of which found the endmember spectra correctly. All of these algorithms generated multiple endmembers corresponding to the same class or they completely missed some of the endmembers. Hence, the peak-aware N-FINDR algorithm was devised to group the endmembers of the same class so as not to over or under-estimate the true endmembers. The comparisons with or without this refinement for the N-FINDR algorithm are demonstrated.
\end{abstract}

Keywords: Endmember extraction algorithm (EEA), hyperspectral unmixing, N-FINDR, initialization.

\section{INTRODUCTION}

In hyperspectral images, pixels are composed of pure materials which are called endmembers. If the spectra of the endmembers is not known beforehead, unmixing of a hyperspectral image requires two stages. These are (1) estimating the endmembers' spectra and (2) calculating their abundance at each pixel. These two stages also require to estimate the number of endmembers before or during unmixing. However, estimating the number of endmembers and their spectrum is a challenging task. For one, endmember detection algorithms may over or underestimate the number of endmembers in a given scene. Further, even if the number of endmembers are known beforehand, result of the endmember detection algorithms may not be accurate. They may find multiple endmembers representing the same class, while completely missing some of the endmembers representing the other classes. This hinders the performance of unmixing, resulting in incorrect endmember proportion estimates. The most popular endmember extraction methods, such as PPI, ${ }^{1} \mathrm{VCA},{ }^{2}$ N-FINDR ${ }^{3}$ and $\mathrm{ICE}^{4}$ all suffer from this problem. Solutions may include feeding the number of endmembers manuall, estimating the number of endmembers beforehand ${ }^{5}$ or estimating the number of endmembers as a joint process of finding the endmembers. ${ }^{6}$ The work focuses on the N-FINDR algorithm for finding the endmembers in a hyperspectral image, and an endmember grouping method, called the peak-aware N-FINDR, has been developed to group similar endmembers to arrive at the best unmixing results.

SHARE-2012 AVON data pertaining to the Unmixing Experiment is used after cropping it to include only the eight pieces of cloth and portion of the surrounding asphalt and grass. Types of these fabrics are blue felt, blue cotton, yellow felt, yellow cotton, pink felt and gold felt as explained in Ref. ${ }^{7}$ and shown in the aerial view in Figure 1. Six of these targets are pure fabrics and their sizes are $\left(12^{\prime} \times 12^{\prime}\right)$. One of the other targets is of size $\left(24^{\prime} \times 24^{\prime}\right)$ which is composed of blue cotton and blue felt materials. Given the approximate $1 \mathrm{~m}$ ground resolution of ProSpecTIR-VS airborne hyperspecral imager, no matter how the pixels are aligned with the target pattern, this arrangement ensures that every pixel in the blue felt/blue cotton material is composed of \%50 blue felt and $\% 50$ blue cotton materials. The last target is of size $\left(16^{\prime} \times 16^{\prime}\right)$ and is composed of yellow felt and yellow cotton 
materials. This target is made with $2 \times 2$ pattern composed of 3 yellow felt squares and 1 yellow cotton square. In doing so, every pixel in this target is composed of $\% 75$ yellow felt and $\% 25$ yellow cotton materials.

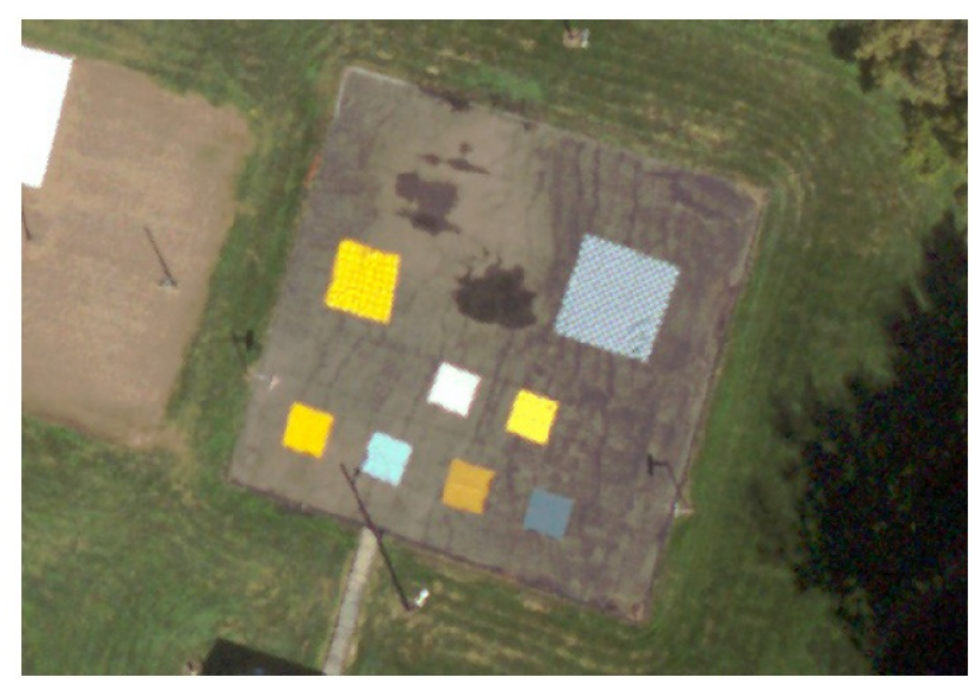

Figure 1: Aerial view of the Share-2012 unmixing experiment. The large $\left(24^{\prime} \times 24^{\prime}\right) \% 50 / \% 50$ blue target is visible in the upper right area of the asphalt court while the smaller $\left(16^{\prime} \times 16^{\prime}\right)$ yellow felt/yellow cotton target on the upper left. The six $\left(10^{\prime} \times 10^{\prime}\right)$ panels of whole colored fabrics are located just below the unmixing targets. The two small panels just below and to the left of the blue target are pink felt (on the left) and yellow cotton (on the right). The four small panels across the lower part of the asphalt are (from left to right): yellow felt, blue cotton, gold felt and blue felt. The image is extracted from RIT WASP imagery collected at 1551Z with approximately 6' resolution Ref. ${ }^{7}$

For testing purposes, first, the original ground truth spectra (OG) and the a manually chosen ground truth spectra (MCG) were compared. The OG had been obtained from a spectrometer measurement, and was provided with the dataset. To make its range comparable to the aerial hyperspectral data, it was interpolated to the spectral range of the hyperspectral data. The MCG on the other hand, was manually chosen from the hyperspectral dataset and from the center of the clothes, which are assumed to represent the pure pixels. The differences between these two ground-truth spectral measurements are shown in Fig. 2.

If, without any processing, the hyperspectral data is classified based on the SAM distances to these groundtruth spectral measurements, the classification results are shown in Fig. 3a for OG and Fig. 3b for MCG. Due to these differences as shown in Fig. 2, MCG spectra was used for the rest of the paper.

In Section 3 our algorithm is explained in detail and test results are presented. In Section 4 hyperspectral image is unmixed using the endmembers found in Section 3 and unsupervised classification is applied to the hyperspectral image. Finally in Section 5 numerical performance of the devised algorithm is presented.

\section{ENDMEMBER DETECTION AND N-FINDR}

Linear mixing model based algorithms are categorized to two main categories. These are Pure Pixel (PP) and Minimum Volume (MV). The algorithms PPI, VCA, N-FINDR are PP based algorithms and these algorithms assume that there is at least one pure pixel which represents each endmember. ${ }^{8}$ SPICE is an example of MV based algorithm. MV based algorithms search for a matrix $\mathbf{M}$ that minimizes the volume of simplex defined by its columns. On the contrary of PP based algorithms, MV based algorithms do not have the constraint that there should be at least one pure pixel representing an endmember.

Among these algorithms, N-FINDR is an algorithm that gives physically understandable results by finding the pixels that that construct a simplex with maximum volume. The pixels which have the maximum volume 


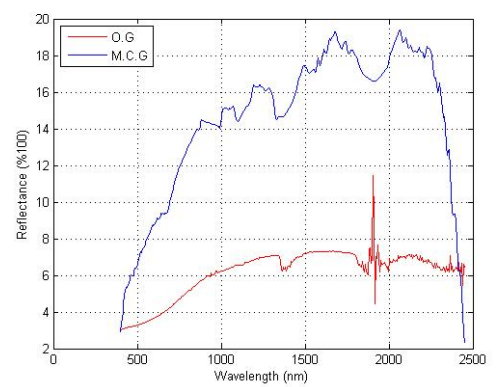

(a) Asphalt

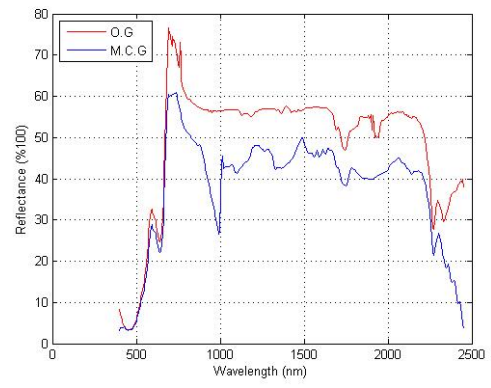

(d) Gold Felt

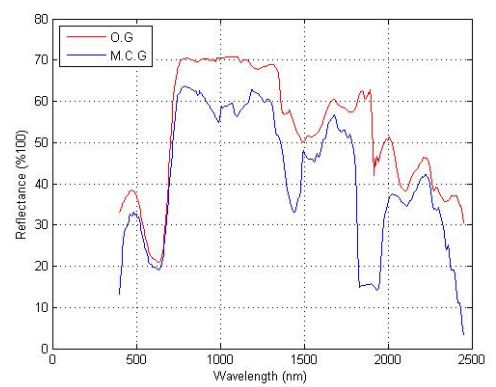

(b) Blue Cotton

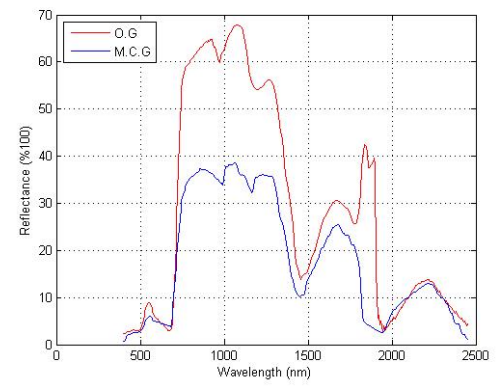

(e) Grass

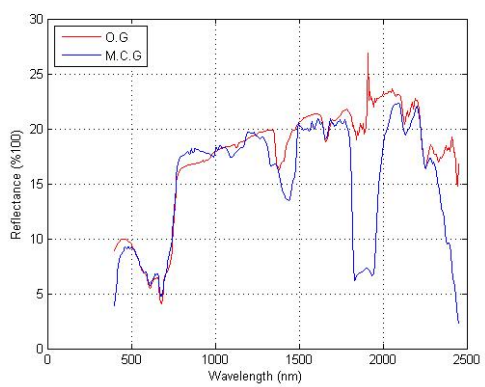

(c) Blue Felt

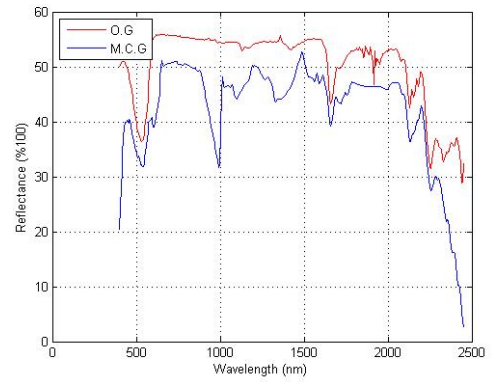

(f) Pink Felt

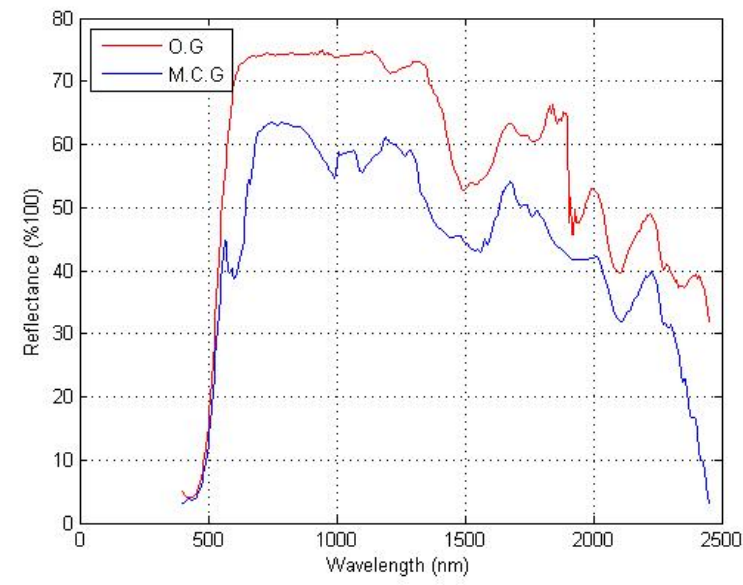

(g) Yellow Cotton

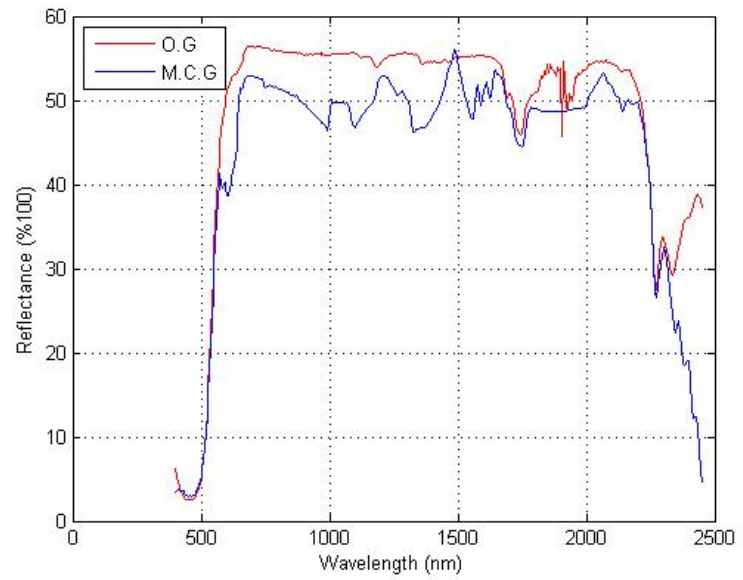

(h) Yellow Felt

Figure 2: Comparison of original ground truth spectra (OG) and the manually chosen ground truth spectra (MCG in blue). OG, in red, are the measurements taken from the ground using a spectrometer and interpolated in the spectral range of original hyperspectral data. MCG, in blue, are chosen from the aerial hyperspectral data, from pure pixels. For clothes, MCG are chosen from center of the material.

are assumed to be endmembers. It is based on the fact that, the volume defined by the purest pixels are larger than any other volume defined by other pixels. As an alternative to the original N-FINDR algorithm, an improved N-FINDR algorithm ${ }^{5}$ was proposed by Antonio Plaza and Chein-I Chang. There are two main differences between the original N-FINDR and the improved N-FINDR algorithms. These are, first, in the original N-FINDR algorithm, the number of endmembers in the image is given to the algorithm externally, whereas in the improved N-FINDR algorithm, number of endmembers are estimated by a criterion called virtual dimensionality (VD). ${ }^{11}$ The second difference is that in the original N-FINDR algorithm, initial endmembers are chosen randomly, whereas improved N-FINDR algorithm uses iterative error analysis ${ }^{12}$ (IEA) algorithm to find 


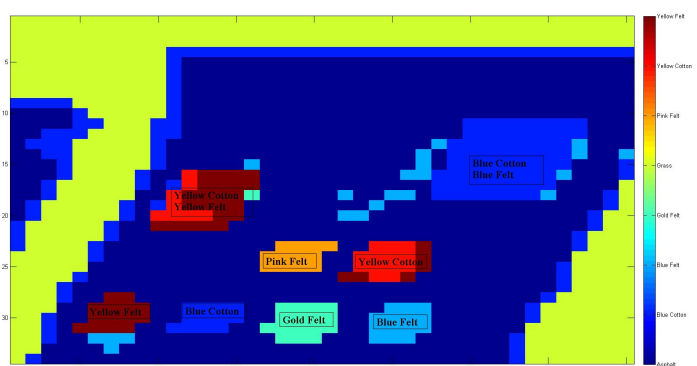

(a) OG Classification

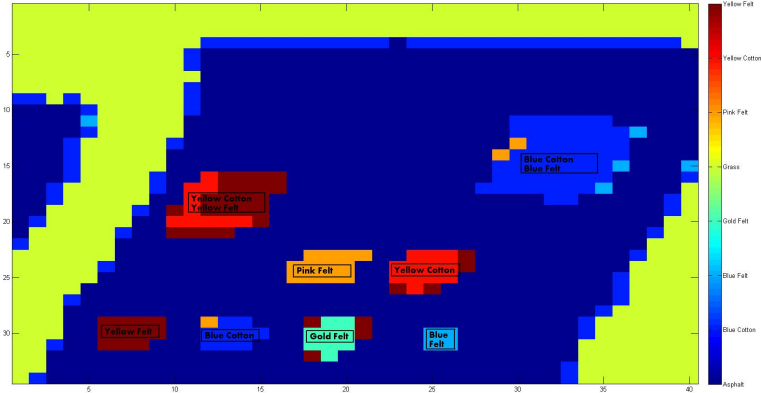

(b) MCG Classification

Figure 3: Hyperspectral image classification results. In (a) hyperspectral image classified using OG and in (b) hyperspectral image is classified using MCG. Classification is performed by computing the SAM distance between pixels spectra and the ground truth spectra. Pixels are classified belonging to the class of ground truth with minimum SAM distance.

the initial endmembers.

N-FINDR algorithm's stages are as follows:

\section{Dimension Reduction}

Hyperspectral image dimension is reduced from $(L)$ to $(p-1)$ using $\mathrm{PCA}^{9}$ or MNF transform. ${ }^{10}(L)$ and $(p)$ corresponds to the original spectral dimension of hyperspectral image and the number of endmembers in the image respectively.

\section{Initialization}

From the hyperspectral image, $p$ pixels are chosen randomly to generate $\left(e_{1}^{(0)}, e_{2}^{(0)}, \ldots, e_{p}^{(0)}\right)$.

\section{Volume Calculation}

At each $k \geq 0$ iteration, volume of endmembers $\left.\mathrm{V}\left(e_{1}^{(k)}, e_{2}^{(k)}, \ldots, e_{p}^{(k)}\right)\right)$ is calculated as follows:

$$
\left.V\left(e_{1}^{(k)}, e_{2}^{(k)}, \ldots, e_{p}^{(k)}\right)\right)=\frac{\left|\operatorname{det}\left[\begin{array}{cccc}
1 & 1 & \cdots & 1 \\
e_{1}^{(k)} & e_{2}^{(k)} & \cdots & e_{p}^{(k)}
\end{array}\right]\right|}{(p-1) !}
$$

\section{Endmember Update}

At the $k$ th stage, each hyperspectral image pixel $r_{i}$ is replaced with each individual endmember and the new simplex volume $\left.\mathbf{V}\left(r, e_{2}^{(k)}, \ldots, e_{p}^{(k)}\right), \mathbf{V}\left(e_{1}^{(k)}, r, \ldots, e_{p}^{(k)}\right), \ldots \mathbf{V}\left(e_{1}^{(k)}, \ldots, e_{(p-1)}\right)^{(k)}, r\right)$ is calculated. If none of these $p$ calculated volumes are greater than $\left.\mathrm{V}\left(e_{1}^{(k)}, e_{2}^{(k)}, \ldots, e_{p}^{(k)}\right)\right)$ than no endmembers are replaced. Otherwise, pixel $r_{i}$ is replaced with $e_{j}^{(k)}$ where $\mathrm{V}\left(e_{1}^{(k)}, e_{2}^{(k)}, \ldots, e_{j}^{(k)}, \ldots, e_{p}^{(k)}\right) \geq \mathrm{V}\left(e_{1}^{(k)}, e_{2}^{(k)}, \ldots, e_{j}^{(k)}, \ldots, e_{p}^{(k)}\right)$. Endmember update stage is repeated until no more replacements are needed.

\section{PEAK-AWARE N-FINDR}

In this study, we introduce an improvement to N-FINDR, the peak-aware N-FINDR, that groups potential endmembers based on similarity, and chooses a representative endmember to represent that group. In doing so, we have observed increased rates of finding the pure spectral pixels; which also propagates to performing better abundance estimation.

In the peak-aware N-FINDR algorithm, we first find 3 times the number of endmembers in the image. In doing so, we try to assure that at least one endmember which represents each class is found. On the flip 
side, this results in finding more than one endmember to represent the same class. To overcome this problem, endmembers' spectra are compared with each other using SAM. If SAM distance between two endmembers is less than a threshold, then these two endmembers are grouped together. Therefore, this stage of the algorithm results in endmember groups.

The next stage of the algorithm is to find one representative endmember from each endmember group. How to choose the endmember which represents its endmember group is another problem. We tested three methods to choose the best representative endmember: (1) choosing the endmember which has peak spectra in all bands, (2) choosing maximum average spectra in all bands and (3) choosing endmember whose absence effects the N-FINDR volume most. In our experiments, we got the best results by choosing the endmembers which has peak spectra in all bands; an idea which also best fits the linear mixture model. Hence, all endmember detection stages can be summarized as follows:

1. Find three times $(3 \times p)$ the endmembers in the image where $p$ is the number of endmembers in the image.

2. Group these endmembers using SAM. Get k endmember groups.

3. Choose $\mathrm{k}$ representative endmembers from each endmember group.

Using the N-FINDR algorithm, 25 endmembers were extracted from the hyperspectral image. These endmembers were grouped using SAM and one representative endmember was chosen from each endmember group. SAM threshold was choosen to be $10^{\circ}$. Location of these ungrouped endmembers is given in Fig. 4a. After the grouping stage, the color-coded group of endmembers are given in Fig. 4b; and the location of the endmembers which represent each endmember group is given in Fig. 4c. Spectra of the each 8 endmember group and 8 endmembers which represent each endmember group is given Fig. 5.

\section{UNMIXING AND CLASSIFICATION RESULTS OF THE SHARE-2012 HYPERSPECTRAL DATASET}

Hyperspectral image is unmixed using the representative endmembers with quadratic programming for the linear mixture model. The linear mixture model has two constraints: (1) for all pixels sum of endmembers' proportion values are equal to one and (2) all proportion values are greater than zero; as given in Equation (2):

$$
\sum_{k=1}^{M} p_{i k}=1, \quad p_{i k} \geq 0, \quad k=1, \ldots ., N
$$

For quadratic programming, the following code was used: "http://sigpromu.org/quadprog". The hyperspectral unmixing results are presented in Fig. 6; where successful abundance estimates were obtained especially for materials with pure pixels.

Following unmixing, unsupervised classification is applied to the hyperspectral image. Two methods were used to classify the image. In the first methods, SAM distance was computed between the pixels and the endmembers. In the second method, unmixing results of quadratic programming were used. First method is as follows;

1. Calculate the SAM difference between pixels and the endmembers.

2. Classify the pixel to belong to the endmember group with the minimum SAM distance.

and the second method is as follows;

1. Hyperspectral image is unmixed using the endmembers found as representative.

2. Classify the pixel to belong to the endmember group which has maximum proportion value. 


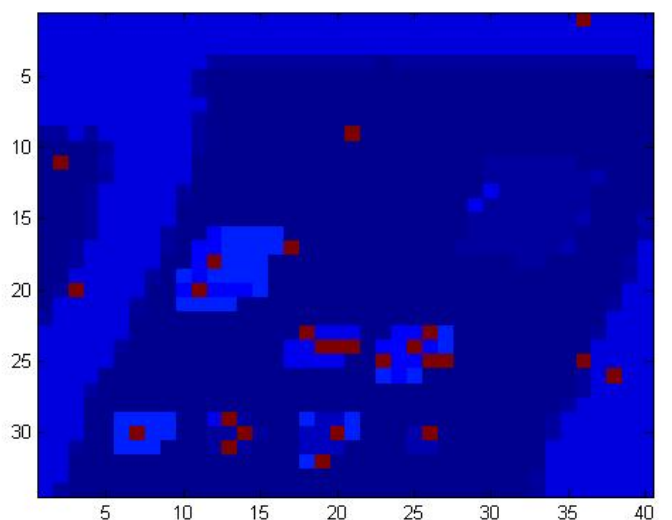

(a) Unmerged Endmembers

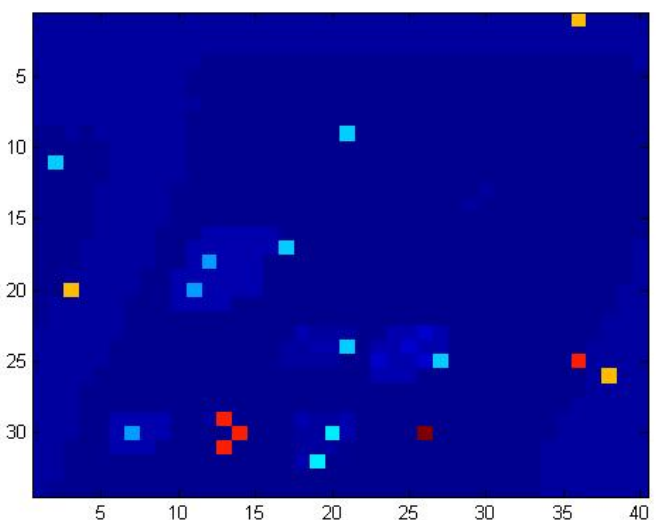

(b) Merged Endmembers

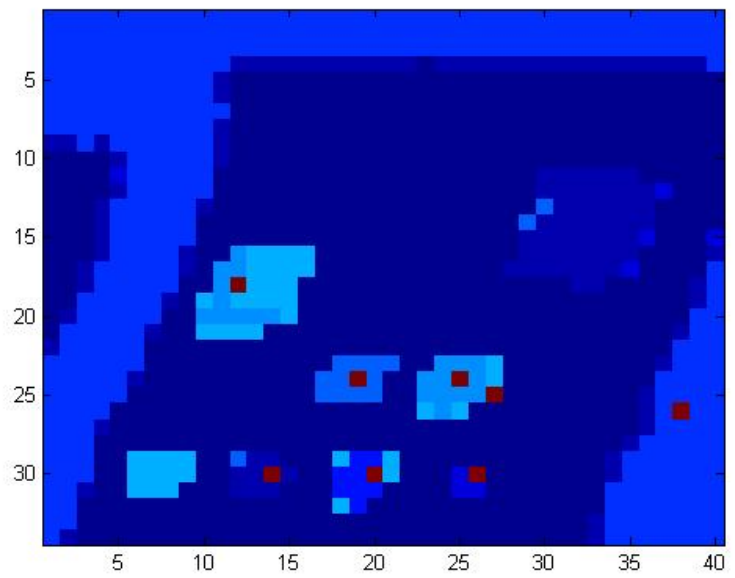

(c) Representative Endmembers

Figure 4: Peak-aware N-FINDR results. 25 endmembers are found in the test site using N-FINDR. Physical locations of these endmembers are given in (a). Endmembers are grouped using the SAM distance. After the grouping process, results are given in (b). Every color group represents an endmember group. From every endmember group, one representative endmember is chosen which has peak spectra along all the bands in its corresponding group. Physical locations of these representative endmembers are given in (c).

Using both of these methods, hyperspectral image was classified using the endmembers found as representatives in Section 3. Classification using unmixing results is given in Fig. 7a and classification using SAM distances between the pixels and the endmembers is given in Fig. 7b. As it can be seen from the classification results, SAM classification results are more definite. On the other hand, unmixing (abundance-based) classification brings the advantage of showing the transition between pure pixels and subpixels.

\section{RESULTS AND CONCLUSION}

We run the devised algorithm 100 times and results are as follows:

1. In 43 out of 100 runs, we found the representative endmembers as representing each of the 8 endmembers.

2. In 94 out of 100 runs, we found the representative endmembers as representing at least 7 endmember groups. 


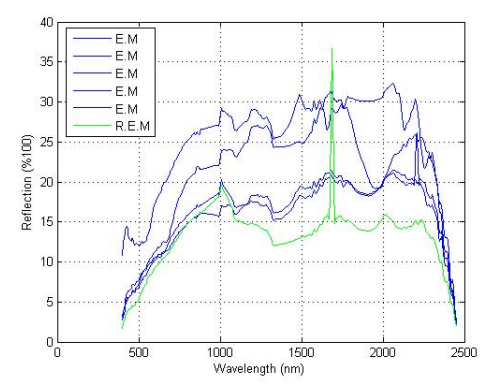

(a) Asphalt

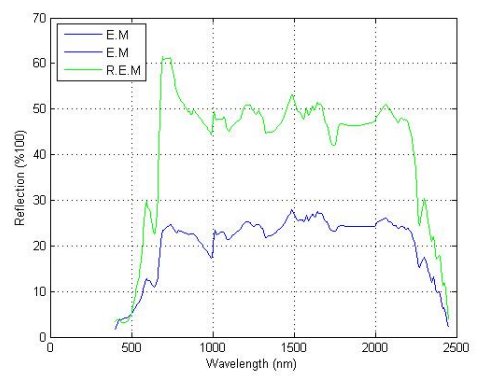

(d) Gold Felt

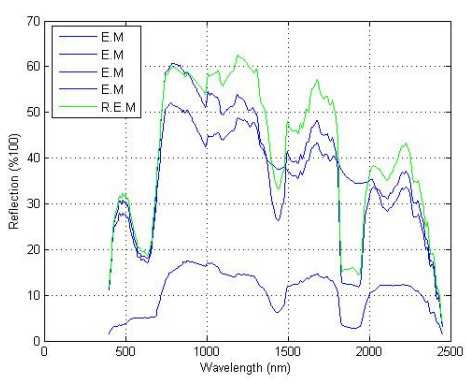

(b) Blue Cotton

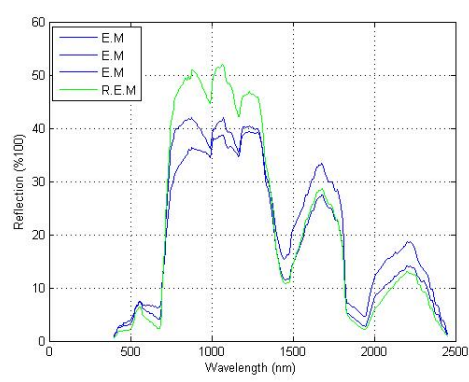

(e) Grass

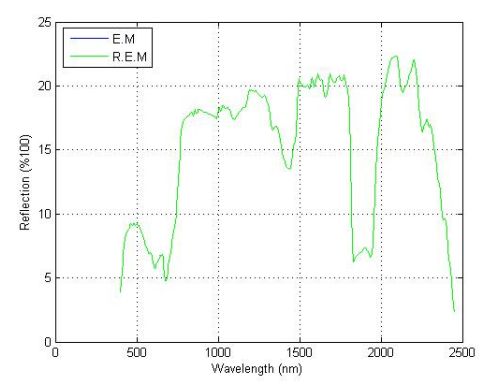

(c) Blue Felt

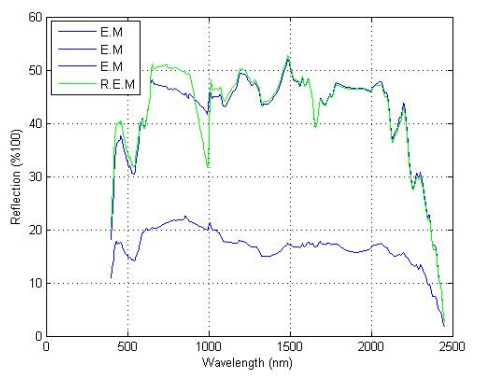

(f) Pink Felt

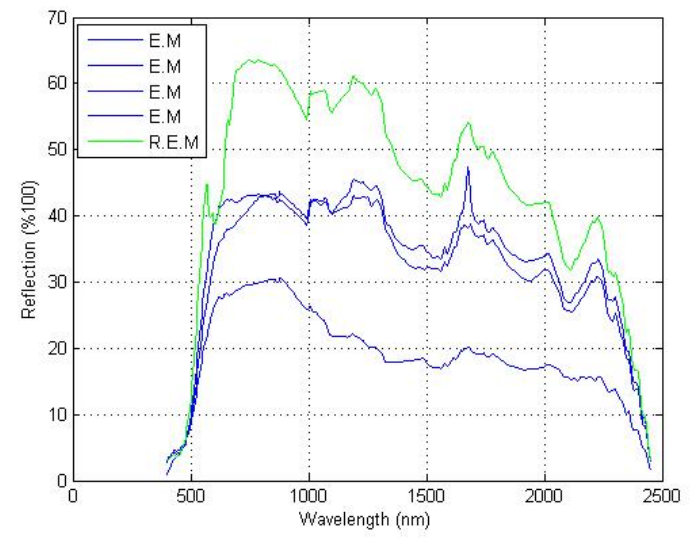

(g) Yellow Cotton

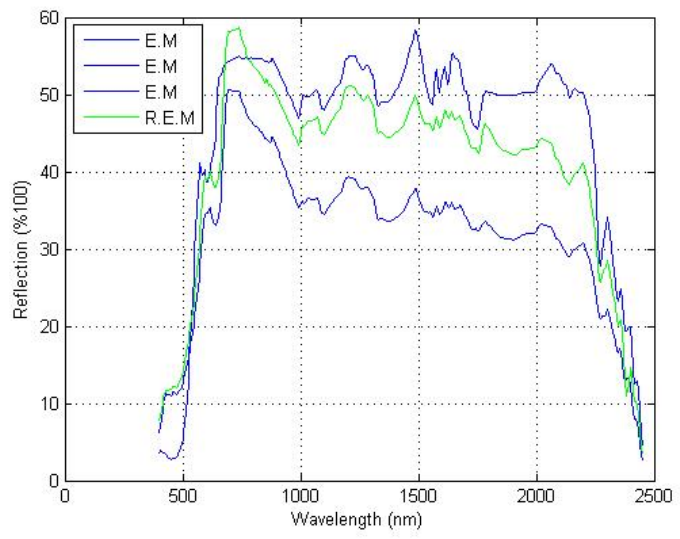

(h) Yellow Felt

Figure 5: Endmember groups found in hyperspectral image. (EM):Potential endmembers are shown in blue, (REM): the representative endmember is shown in green. Three methods are tried to find best REM. These are (1) choosing endmember which has peak spectra in all bands, (2) choosing endmember which has maximum average spectra in all bands and (3) choosing endmember which absence effects N-FINDR volume most. Because of choosing endmembers which has peak spectral in all bands best fits linear mixture model, we use method (1) for choosing REM. 


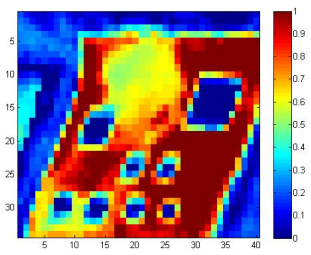

(a) Aspalt

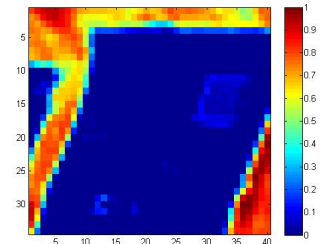

(e) Grass

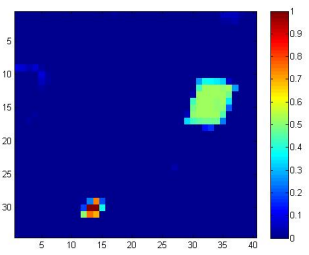

(b) Blue Cotton

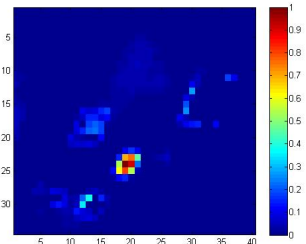

(f) Pink Felt

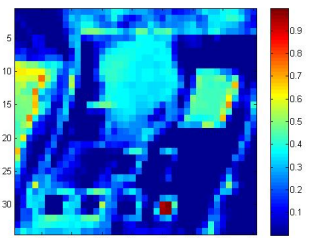

(c) Blue Felt

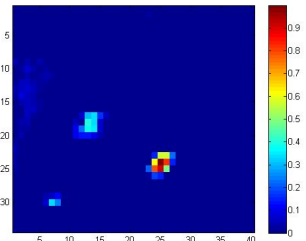

(g) Yellow Cotton

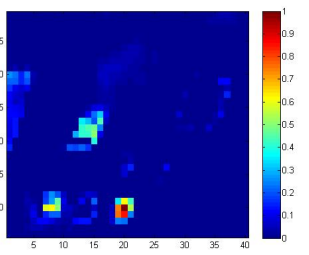

(d) Gold Felt

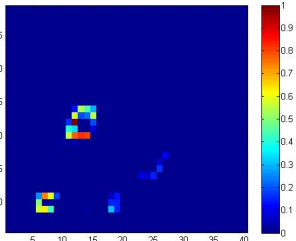

(h) Yellow Felt

Figure 6: Results of hyperspectral unmixing. Unmixing is performed with the representative endmembers that each one represent unique pure material in the hyperspectral image.

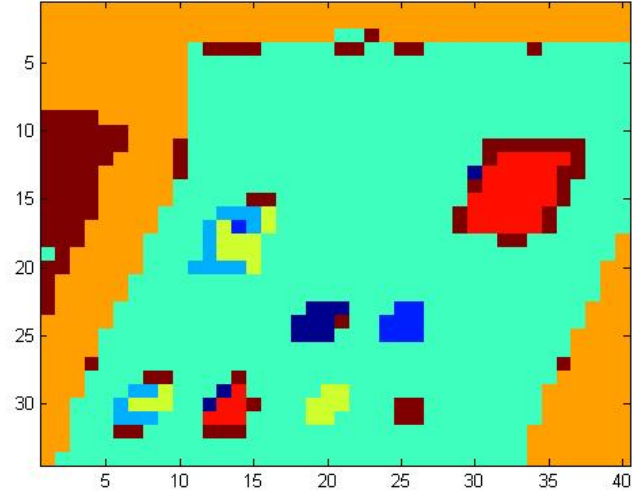

(a) Unmixing Result Classification

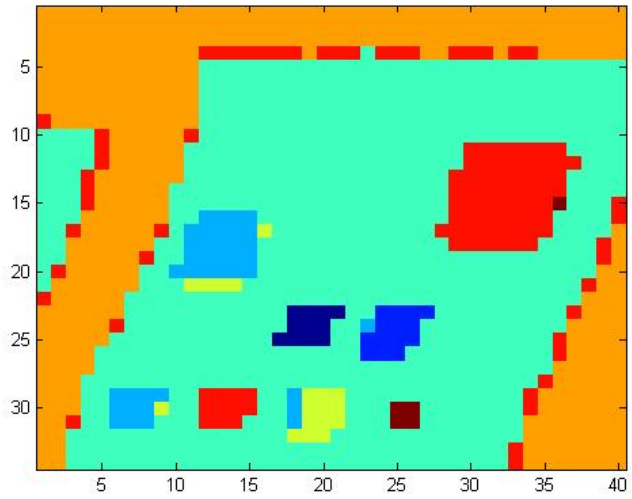

(b) SAM Classification

Figure 7: Hyperspectral image classification result. Classification result using SAM distance between pixels and endmembers is given in (a). Classification result using hyperspectral image unmixing is given in (b).

For unmixing experiment, we chose 10 pixels to test the overall devised algorithm and the quadratic programming algorithm. 8 of these pixel are pure and the 2 of these pixels are subpixels. Location of the pure pixels are given in Fig. 8a and the location of subpixels are in given Fig. 8b. Each of these 8 pure pixels represent one pure endmember in the image and one of these subpixels represent blue cotton/blue felt, the other one represents yellow cotton/yellow felt subpixel.

Each of these found endmembers are tested on related test pixels. That means, asphalt endmember is tested on asphalt test pixel and overall number of found asphalt endmembers and unmixing result of asphalt endmember on asphalt test pixel is recorded. Asphalt, grass, gold felt, pink felt endmembers are only tested on pure pixels. Blue cotton, blue felt, yellow cotton and yellow felt endmembers are both tested on pure pixels and subpixels. Pure pixels are given in Table 1. The result of mean and standard deviation are calculated for the representative endmembers found by peak-aware N-FINDR. Original mean and standart deviation of the subpixels given with the dataset and the experimental subpixel results are given in Table 2.

To conclude, there are two main factors that effect the unmixing results of the hyperspectral image. The first one is finding the endmembers that represent each pure material. In this work, we found multiple times the number of endmembers in the hyperspectral image. The disadvantage is that some of the found endmembers 


\begin{tabular}{|c|l|l|l|}
\hline Endmembers & $\begin{array}{l}\text { The number of } \\
\text { times endmembers } \\
\text { are found on the } \\
\text { related test pixel }\end{array}$ & $\begin{array}{l}\text { Mean of the abun- } \\
\text { dances found on the } \\
\text { related test pixel }\end{array}$ & $\begin{array}{l}\text { Standard deviation } \\
\text { of abundances } \\
\text { found on related } \\
\text { test pixel }\end{array}$ \\
\hline Asphalt & $82 / 100$ & 0.81 & 0.16 \\
\hline Blue Cotton & $100 / 100$ & 0.91 & 0.16 \\
\hline Blue Felt & $69 / 100$ & 0.97 & 0.02 \\
\hline Gold Felt & $90 / 100$ & 0.89 & 0.12 \\
\hline Grass & $100 / 100$ & 0.71 & 0.07 \\
\hline Pink Felt & $100 / 100$ & 0.99 & 0.05 \\
\hline Yellow Cotton & $100 / 100$ & 0.90 & 0.16 \\
\hline Yellow Felt & $59 / 100$ & 0.89 & 0.16 \\
\hline
\end{tabular}

Table 1: Pure Pixel Results

\begin{tabular}{|l|l|l|l|l|}
\hline & Yellow Felt & $\begin{array}{l}\text { Yellow Cot- } \\
\text { ton }\end{array}$ & Blue Felt & Blue Cotton \\
\hline True Fractions & 0.75 & 0.25 & 0.50 & 0.50 \\
\hline 3 Guard Pixels & $0.77 \pm 0.016$ & $0.26 \pm 0.005$ & $0.52 \pm 0.007$ & $0.53 \pm 0.021$ \\
\hline Test Results & $0.66 \pm 0.27 ;$ & $0.30 \pm 0.11 ;$ & $0.41 \pm 0.04 ;$ & $0.49 \pm 0.09 ;$ \\
& NE: $48 / 100$ & NE:72/100 & NE:69/100 & NE:99/100 \\
\hline
\end{tabular}

Table 2: The deviation from the fractions provided with the dataset. Retrieved fractions of materials found using unconstrained spectral unmixing applied to the Yellow/Yellow and Blue/Blue unmixing targets. The mean and standart deviations are from the set of unmixed fractions from pixels in the target area. This informations can be found in Ref. ${ }^{7}$ Test results are the experimental subpixel results.

are not pure spectra and we found more than one endmember representing the same class. To overcome this problem we grouped the endmembers using SAM and chose representative endmembers which has peak spectra in all bands. Another factor that effect the endmember detection results is, the method we used compare the endmember spectra. The second factor which effects the result is the algorithm used for unmixing. In this work we used quadratic programming to unmix the hyperspectral image. Even if the MCG spectra are given to the algorithm as endmembers, we could not get the unmixing results of pure/subpixels as it given with the dataset. Most of the endmember extraction algorithm devised for finding the endmembers in the hyperspectral image, generate multiple endmembers corresponding to the same class. Unmixing the hyperspectral image with the endmembers corresponding to the same class cause error. Devised algorithm's first stage may be skipped for the case that there are not multiple times the endmember corresponding to the same class. The devised algorithm's second and third stages should be performed before hyperspectral image is unmixed.

\section{REFERENCES}

1. J. Boardman, "Automating spectral unmixing of aviris data using convex geometry concepts," Proc. Ann. JPL Airborn Geosci. 1, pp. 11-14, 1993.

2. J. M. P. Nascimento and J. M. Bioucas-Dias, "Vertex component analysis: A fast algorithm to unmix hyperspectral data," IEEE Trans. Geosci. Remote Sens. 43, pp. 898-910, 2005.

3. M. E. Winter, "N-findr: An algorithm for fast autonomous spectral endmember determination in hyperspectral data," Proc. SPIE Image Spectrometry V 3753, pp. 266-277, 1999.

4. M. Berman, H. Kiiveri, R. Lagerstrom, A. Ernst, R. Dunne, and J. F. Huntington, "Ice: A statistical approach to identifying endmembers in hyperspectral images," IEEE transactions on Geoscience and Remote Sensing 42(10), pp. 2085-2095, 2004.

5. A. Plaza and C.-I. Chang, "An improved n-findr algorithm in implementation," Algorithm and Technologies for Multispectral, Hyperspectral and Ultraspectral Imagerry XI . 


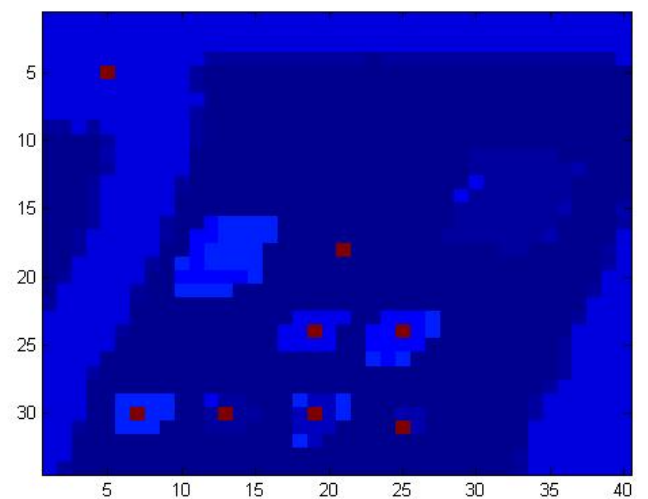

(a) Pure Pixels

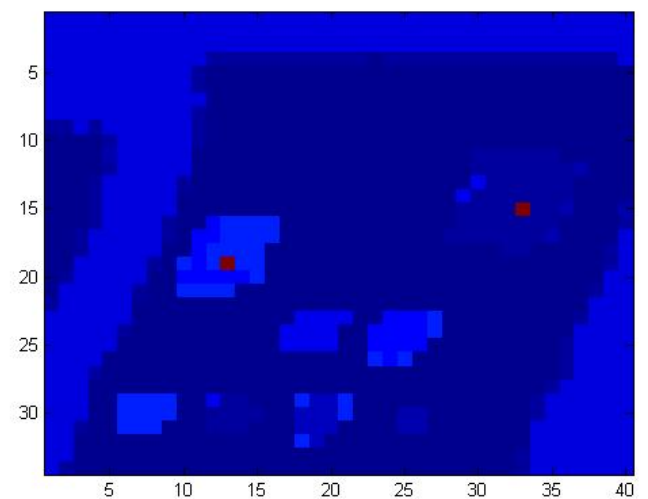

(b) Sub Pixels

Figure 8: Location of test pixels. Table 1 is obtained by running the algorithm on the pure pixels shown in (a). Table 2 is obtained by running the algorithm on the subpixels shown in (b).

6. A. Zare and P. D. Gader, "Sparsity promoting iterated constrained endmember detection for hyperspectral imagery," IEEE Geosci. Remote Sens. Lett. 4 no. 3, pp. 466-450, 2007.

7. J. P. Kerekes, K. Ludgate, A. Giannadrea, and a. D. S. G. Nina G. Raqueno, "Share 2012: Subpixel detection and unmixing experiment,"

8. J. M. Bioucas-Dias, A. Plaza, M. P. Nicolas Dobigeon, Q. Du, P. Gader, and J. Chanussot, "Hyperspectral unmixing overview: Geometrical, statistical, and sparse regression-based approaches," IEEE Journal Of Selected Topics In Applied Earth Observations and Remote Sensing 5 no. 2, 2012.

9. I. T. Jolliffe, "Principal component analysis," New York: Springer Verlag, 1988.

10. A. A. Green, M. Berman, P. Switzer, and M. D. Craig, "A transformation for ordering multispectral data in terms of image quality with implications for noise removal," IEEE Trans. Geosci. Remote Sens 26 , p. 6574.

11. A. Plaza and C.-I. Chang, "Hyperspectral imaging: Techniques for spectral detection and classification," Kluwer Academic/Plenum Publishers, 2003.

12. R. Neville, K. Staenz, T. Szeredi, J. Lefebvre, and P. Hauff, "Automatic endmember extraction from hyperspectral data for mineral exploration," 4th International Airborne Remote Sensing Conf. and Exhibition/21st Canadian Symposium on remote Sensing, p. 2124. 